\title{
Fast Heuristics for Eliminating Switching Components in Binary Matrices by 0-1 Flips*
}

\author{
Norbert Hantos and Péter Balázs \\ Department of Image Processing and Computer Graphics \\ University of Szeged \\ Árpád tér 2. H-6720, Szeged, Hungary \\ \{nhantos,pbalazs\}@inf.u-szeged.hu
}

\begin{abstract}
Switching components are special patterns in binary matrices that play an essential role in many image processing and pattern analysis tasks. Finding the minimal number of 0 s that must be switched to $1 \mathrm{~s}$ in order to eliminate all switching components is an NP-complete problem. We present two novel-type heuristics for the above problems and show via experiments that they outperform the formerly proposed ones, both in optimality and in running time.
\end{abstract}

Keywords: binary matrix, image reconstruction, switching component, uniqueness, 0-1 flip.

\section{Introduction}

Analysis of binary patterns plays a vital role in numerous applications of computer science such as intelligent data analysis [1], data mining [8, low-level image processing [3, and machine learning [6]. Binary patterns can be naturally encoded by binary matrices, and one commonly performed task is to localize and enumerate special subpatterns in that matrix. Switching components are $2 \times 2$ submatrices with exactly two $1 \mathrm{~s}$ in the diagonal and two $0 \mathrm{~s}$ in the antidiagonal (or vice versa), that play an important role in discrete image reconstruction 4. The absence of switching components in the matrix is a necessary and sufficient condition for the unique reconstruction of the matrix from the vectors of its row and columns sums. If uniqueness is guaranteed then the binary image represented by the binary matrix can be stored in a (lossless) compressed form

\footnotetext{
^ The work of Péter Balázs was supported by the OTKA PD100950 project of the National Scientific Research Fund and by the European Union and the State of Hungary, co-financed by the European Social Fund (TÁMOP-4.2.4.A/2-11-1-2012-0001, "National Excellence Program"). The research of Norbert Hantos was supported by the European Union co-funded by the European Social Fund (TÁMOP-4.2.2.A11/1/KONV-2012-0073, "Telemedicine-focused research activities on the field of Mathematics, Informatics and Medical sciences") and by the Research Group on Artificial Intelligence (RGAI), belonging to the Hungarian Academy of Sciences (MTA) and University of Szeged (SZTE) Informatics Departments.
}

E. Bayro-Corrochano and E. Hancock (Eds.): CIARP 2014, LNCS 8827, pp. 62-69 2014.

(C) Springer International Publishing Switzerland 2014 

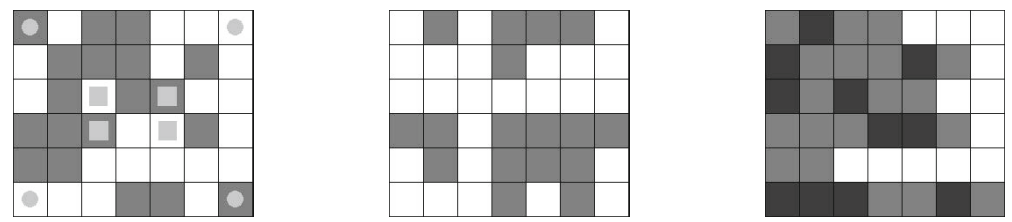

Fig. 1. A binary image with two examples of switching components (left), a switching component free binary image (middle), the canonical expansion of the first image (right)

by those two vectors. Nevertheless, even if the matrix contains switching components, there is still a chance to reconstruct the matrix uniquely, if properly chosen elements of the matrix are stored as well [2]. One can store, e.g., the positions of $0 \mathrm{~s}$ which need to be inverted to $1 \mathrm{~s}$ (by so-called $0-1$ flips) in order to make the matrix switching component free. The aim is then to find the minimal number of 0 -1 flips needed to achieve uniqueness. In biogeography, binary matrices can represent the presence or absence of certain species (rows) on certain locations (columns). Here, the so-called nestedness is a relevant measure of the matrix, which is also strongly connected to the theory of 0-1 flips [5].

Unfortunately, the problem of determining the minimal number of $0-1$ flips to achieve uniqueness is generally NP-complete [5]. In this paper we show that the minimal number of $0-1$ flips can be found by determining the proper ordering of the columns according to a certain filling function, instead of searching through matrix elements and switching components. Based on theoretical results, we develop two deterministic, polynomial-time heuristics to find the minimal number of 0-1 flips. We compare those algorithms to another well-known methods in the literature, on a wide set of random binary matrices. We conclude that the algorithms searching for proper column permutations perform better, both in the number of 0-1 flips and running time, especially on sparse matrices.

This paper is structured as follows. Section 2 is for the preliminaries. In Section 3 we present theoretical results that form the basis of our heuristics. In Section 4 we describe the heuristics in detail. Then, in Section 5 we present the experimental results. Finally, in Section 6] we give the conclusions.

\section{Preliminaries}

Let $A=\left(a_{i j}\right)_{m \times n}$ be a binary matrix. If the value in a position of $A$ is 0 (respectively, 1) then in the image representation of $A$ the corresponding pixel is white (respectively, black). Let $|A|$ denote the number of $1 \mathrm{~s}$ in the binary matrix $A$, hence, $|A|=\sum_{i} \sum_{j} a_{i j}$. We say that the binary matrix $A$ is a subset of the binary matrix $B$, and denote it by $A \subseteq B$, if both matrices have the same size, and for all $(i, j)$ positions whenever $a_{i j}=1$ then $b_{i j}=1$.

The indices $i_{1}, j_{1}, i_{2}, j_{2}$ form a switching component in the binary matrix $A$, if either $a_{i_{1} j_{1}}=a_{i_{2} j_{2}}=1$ and $a_{i_{1} j_{2}}=a_{i_{2} j_{1}}=0$, or $a_{i_{1} j_{1}}=a_{i_{2} j_{2}}=0$ and $a_{i_{1} j_{2}}=a_{i_{2} j_{1}}=1$. A binary matrix is switching component free, if it contains no switching components (see Fig. 1 left and middle images). 
A 0 -1 flip is an operation that changes a 0 element of a given binary matrix to 1 . The canonical expansion of the binary matrix $A$ is a binary matrix with the same size as of $A$, provided by $\psi A$ which is defined as

$$
\psi a_{i j}= \begin{cases}0 & \text { if } a_{i j^{\prime}}=0 \text { for every } j^{\prime} \geq j \\ 1 & \text { otherwise. }\end{cases}
$$

Figure 1 shows an example for a canonical expansion. It is easy to see that $\psi \psi A=\psi A$. Furthermore, $A \subseteq \psi A$, since $\psi$ performs only $0-1$ flips in $A$. If $\psi A=A$ for a binary matrix $A$, then $A$ is a canonical matrix. Since $\psi \psi A=\psi A$, the canonical expansion of any binary matrix is a canonical matrix.

Finally, given the binary matrix $A$ with the size of $m \times n$ and a permutation $\pi$ of order $n$, let $\pi A$ denote the binary matrix which consists of the columns of $A$ according to $\pi$.

\section{Problem Setting and Theoretical Results}

Our goal is to answer the following problem.

\section{Problem. Minimum Flip Augmentation (MFa)}

Description. Given a binary matrix $A$, find a switching component free binary matrix $A^{*}$ such that $A^{*}$ is constructed from $A$ by a minimal number of $0-1$ flips.

Changing each 0 to 1 in $A$ would yield a binary matrix with no switching components, therefore such an $A^{*}$ matrix always exists. However, in [5] the following lemma is proven.

Lemma 1. MFA is NP-complete.

A naive approach to find an (approximate) optimal solution may include a search through the switching components of $A$, and eliminate them by changing a 0 value of them into 1 , in a sequential order. However, the number of switching components can be of $O\left(m^{2} n^{2}\right)$, and an exhaustive search for the optimal solution may require $O((m n) !)$ steps. We show how to speed up the searching process through canonical expansions, in order to gain much faster approximate solutions. In that case, the exhaustive search will require at most $O((\min \{m, n\}) ! \cdot m n)$ steps. Before describing the heuristics methods, we provide some important theoretical results. The following two lemmas show important properties of canonical matrices, as a direct consequence of Ryser's theorem [7].

Lemma 2. Any canonical matrix is switching component free.

Lemma 3. Let $A$ be a switching component free binary matrix with non-increasing column sums, i.e., $\sum_{i} a_{i 1} \geq \sum_{i} a_{i 2} \geq \cdots \geq \sum_{i} a_{i n}$. Then $A$ is a canonical matrix.

The next lemma describes an important property of the canonical expansion. 
Lemma 4. Let $A$ and $B$ be two binary matrices of the same size $m \times n$. If $A \subseteq B$ then $\psi A \subseteq \psi B$.

Proof. Let $i \in\{1, \ldots, m\}$ be an arbitrary row index. Moreover, let $j_{l(A)}$ denote the position of the last 1 in the $i$-th row of $A$, i.e., $a_{i j_{l(A)}}=1$ and $a_{i j}=0$ for $j>j_{l(A)}$. Similarly, let $j_{l(B)}$ denote the position of the last 1 in the $i$-th row of $B$, hence $b_{i j_{l(B)}}=1$ and $b_{i j}=0$ for $j>j_{l(B)}$. From $A \subseteq B$ it follows that $j_{l(A)} \leq j_{l(B)}$. By the definition of the canonical expansion, $\psi a_{j}=1$ if and only if $1 \leq j \leq j_{l(A)}$. Similarly, $\psi b_{j}=1$ if and only if $1 \leq j \leq j_{l(B)}$. Since $j_{l(A)} \leq j_{l(B)}$, it follows that $\psi b_{k}=1$ whenever $\psi a_{k}=1$ for $k=1, \ldots, n$. The row index $i$ was chosen arbitrarily, thus we get $\psi A \subseteq \psi B$.

Finally, the following theorem shows the connection between canonical expansions and the solutions of the MFA problem.

Theorem 1. Let $A$ be a binary matrix of size $m \times n$, and let $A^{*}$ denote a solution of $\operatorname{MFA}(A)$. Then there exists a column permutation $\pi$ of order $n$ such that $\pi^{-1} \psi \pi A=A^{*}$.

Proof. Let $\pi$ be the (not necessarily unique) permutation such that $\pi A^{*}$ is a binary matrix with non-increasing column sums. Formally, $\pi$ is the column permutation with $\sum_{i} \pi a_{i 1}^{*} \geq \sum_{i} \pi a_{i 2}^{*} \geq \cdots \geq \sum_{i} \pi a_{i n}^{*}$.

Trivially, $A \subseteq A^{*}$, and by the definition of the column permutation and the subset relation, $\pi A \subseteq \pi A^{*}$. A column permutation has no effect on the existence of switching components, hence $\pi A^{*}$ is still switching component free. But then, by Lemma $3 \pi A^{*}$ is canonical and therefore $\psi \pi A^{*}=\pi A^{*}$. Since $\pi A \subseteq \pi A^{*}$, by Lemma 4 we get $\psi \pi A \subseteq \psi \pi A^{*}=\pi A^{*}$. Therefore, $\pi^{-1} \psi \pi A \subseteq A^{*}$.

On the other hand, by the definition of canonical expansion, $\pi A \subseteq \psi \pi A$, and therefore, $A \subseteq \pi^{-1} \psi \pi A$. Moreover, on the basis of Lemma $2 \psi \pi A$ is switching component free, thus $\pi^{-1} \psi \pi A$ is also switching component free. Furthermore, from the arguments of the previous paragraph it follows that $\left|\pi^{-1} \psi \pi A\right| \leq\left|A^{*}\right|$, and therefore $\left(\left|\pi^{-1} \psi \pi A\right|-|A|\right) \leq\left(\left|A^{*}\right|-|A|\right)$. Since $A^{*}$ is a solution of the $\operatorname{MFA}(A)$ problem, the right hand side of above inequality is the possible minimum. Therefore the left hand side must be also minimal, thus $\pi^{-1} \psi \pi A$ must be a solution of the $\operatorname{MrA}(A)$ problem. We have that $\left|\pi^{-1} \psi \pi A\right|=\left|A^{*}\right|$ which together with $\pi^{-1} \psi \pi A \subseteq A^{*}$ yields $\pi^{-1} \psi \pi A=A^{*}$.

Figure 2 illustrates Theorem 1. Unfortunately, the proof of the theorem defines $\pi$ as a function of the solution $A^{*}$, and due to Lemma 1 finding the proper column permutation is generally NP-complete. Nevertheless, the number of possible column permutations is much fewer than the number of possible sequences of switching components, in general.

Corollary 1. To find a solution of the MFA(A) problem, it is sufficient to search for the corresponding column permutation $\pi$. The number of such permutations is $O(n !)$, or considering the transposed matrix, $O((\min \{m, n\}) !)$.

Constructing the canonical expansion of a matrix can be done in $O(m n)$ time, and thus an exhaustive search for the optimal $\pi$ permutation requires $O((\min \{m, n\}) ! \cdot m n)$ time, in the worst case. 


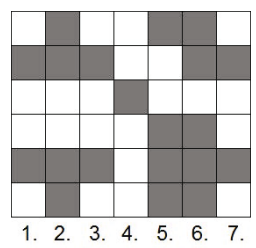

(a) $A$



(d) $\pi A$

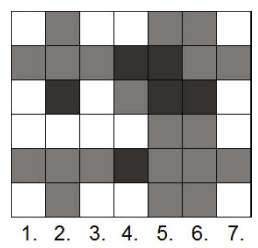

(b) $A^{*}$

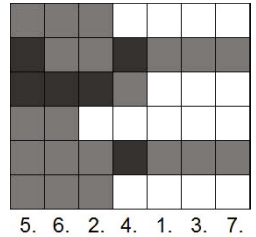

(e) $\psi \pi A$

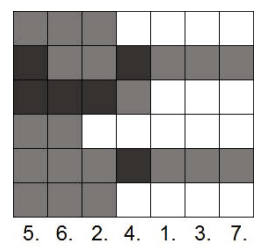

(c) $\pi A^{*}$

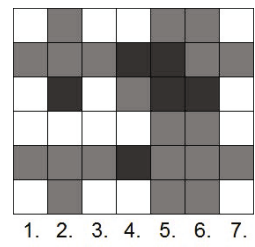

(f) $\pi^{-1} \psi \pi A$

Fig. 2. (a) Binary image $A$, (b) an $A^{*}$ solution of $\operatorname{MFA}(A)$, (c) applying permutation $\pi$ on the columns of $A^{*}$ ensures non-increasing column sums, (d) applying the same column permutation on $A$, (e) the canonical expansion of $\pi A$, (f) and applying the inverse of $\pi$ on the columns of $\psi \pi A$. Note that images (b) and (f) are the same. Numbers indicate the original column indices

\section{Heuristics}

We now describe four different heuristics for the MFA problem, which try to minimize the number of $0 \mathrm{~s}$ need to be flipped to $1 \mathrm{~s}$ in order to make the matrix switching component free. All of them are deterministic methods and have a polynomial running time. Since MFA is NP-complete by Lemma 1, their results naturally cannot be assumed as optimal for all possible input matrices.

Algorithms Switch (Algorithm 1) and Columns (Algorithm 2) are taken from [5] for comparison. SwITCH is a switching component searching algorithm, while Columns works with column permutations. Although [5] does not use the concept of canonical expansions, for technical convenience, we give the pseudo code of Columns to our terms. Our own methods ColPerm1 (Algorithm 3) and ColPERm2 (Algorithm 4) are based on the result of Theorem 1 and Corollary 1. All algorithms require a binary matrix $A$ with the size of $m \times n$, and ensure a binary matrix $A^{\prime}$ such that $A \subseteq A^{\prime}$ and $A^{\prime}$ is switching component free.

\section{$5 \quad$ Numerical Results}

We studied the performance of the algorithms described in Section 4 on random binary matrices. Our test set contained matrices of size $20 \times 20,40 \times 40,60 \times 60$, $80 \times 80$, and $100 \times 100$ and with exactly $10 \%, 20 \%, \ldots, 90 \%$ number of 1 s related to the total number of the matrix entries. With each size and density we generated 50 binary matrices from uniform random distribution. Thus, our 

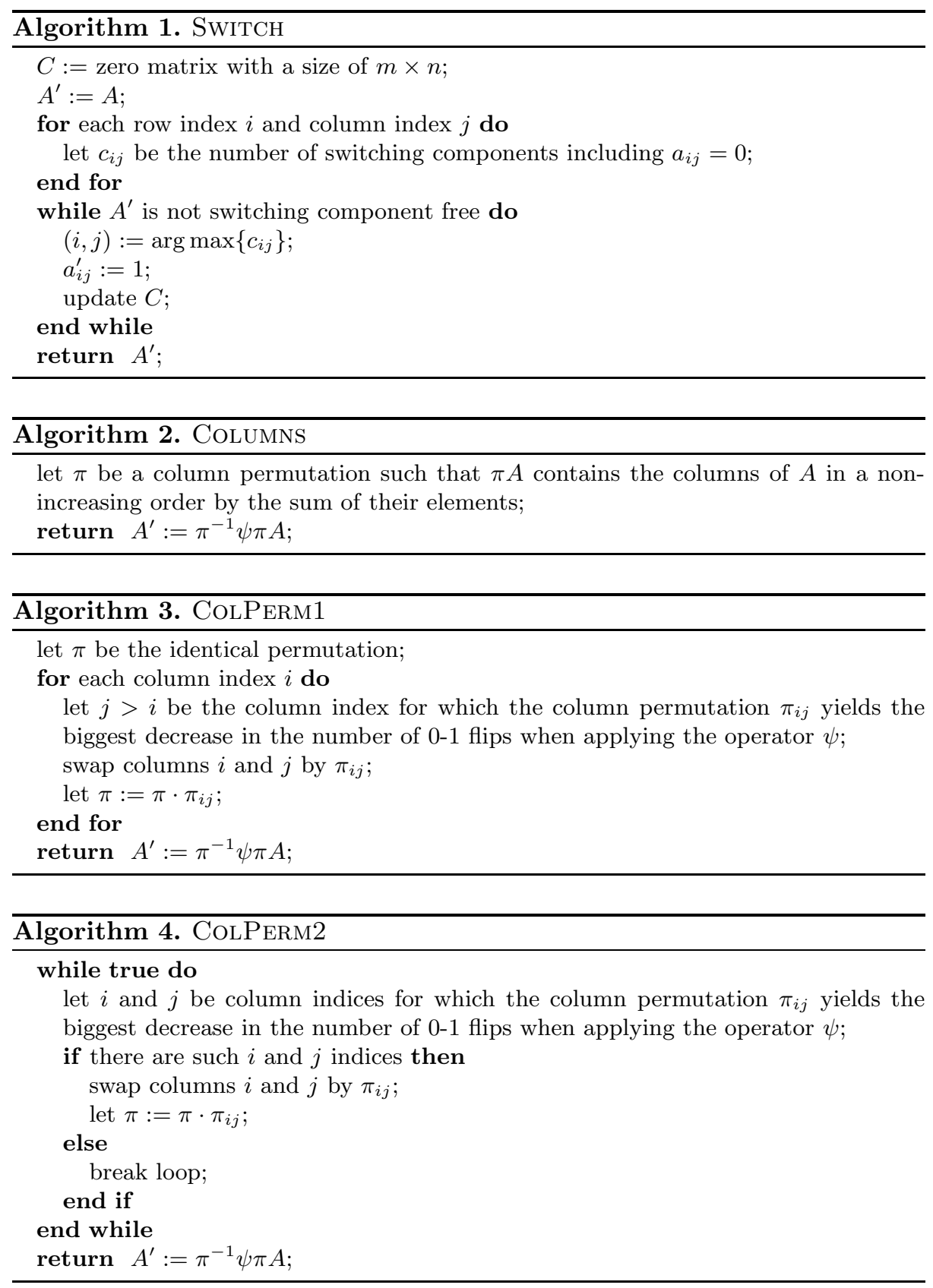

test set consisted of 2250 matrices in total. We implemented the algorithms in MATLAB 7.13.0.564. The test was performed under Windows 7 on one core of an Intel Core i5-2410M of $2.3 \mathrm{GHz}$ PC with $4 \mathrm{~GB}$ of RAM. 
Table 1. Average number of 0-1 flips calculated by the algorithms SwITch (SWI), Columns (COL), ColPerm1 (CP1), and ColPerm2 (CP2)

\begin{tabular}{r|r|r|r||r|r|r|r||r|r|r|r}
\multicolumn{1}{c||}{$20 \times 20$} & \multicolumn{5}{|c||}{$40 \times 40$} & \multicolumn{3}{c}{$60 \times 60$} \\
\hline SWI & COL & CP1 & \multicolumn{1}{|c||}{ CP2 } & SWI & COL & CP1 & \multicolumn{1}{c||}{ CP2 } & SWI & COL & CP1 & \multicolumn{1}{c}{ CP2 } \\
\hline 121.8 & 133.5 & 120.7 & $\mathbf{1 1 4 . 2}$ & 831.9 & 851.3 & 768.3 & $\mathbf{7 2 9 . 4}$ & 2341.5 & 2325.7 & 2088.0 & $\mathbf{2 0 2 4 . 9}$ \\
172.1 & 191.7 & 170.9 & $\mathbf{1 6 3 . 7}$ & 986.2 & 1008.6 & 928.1 & $\mathbf{9 0 0 . 3}$ & 2481.4 & 2475.8 & 2316.7 & $\mathbf{2 2 6 9 . 9}$ \\
175.4 & 200.6 & 180.0 & $\mathbf{1 7 3 . 2}$ & 929.5 & 965.3 & 900.8 & $\mathbf{8 7 9 . 8}$ & 2297.6 & 2283.2 & 2181.7 & $\mathbf{2 1 3 9 . 2}$ \\
$\mathbf{1 6 1 . 6}$ & 183.5 & 168.4 & 162.0 & 823.4 & 854.7 & 811.1 & $\mathbf{7 9 7 . 1}$ & 2003.8 & 2012.5 & 1940.0 & $\mathbf{1 9 1 5 . 2}$ \\
$\mathbf{1 4 5 . 7}$ & 162.6 & 151.3 & 147.8 & 695.0 & 729.1 & 698.9 & $\mathbf{6 8 7 . 8}$ & 1684.4 & 1702.1 & 1652.4 & $\mathbf{1 6 3 4 . 3}$ \\
$\mathbf{1 2 1 . 8}$ & 136.0 & 127.2 & 125.0 & $\mathbf{5 6 3 . 0}$ & 592.8 & 570.8 & 564.0 & 1343.2 & 1372.8 & 1338.3 & $\mathbf{1 3 2 7 . 5}$ \\
$\mathbf{9 1 . 3}$ & 102.3 & 95.7 & 93.9 & $\mathbf{4 2 3 . 0}$ & 447.7 & 433.4 & 427.8 & $\mathbf{1 0 0 0 . 6}$ & 1036.2 & 1011.4 & 1005.4 \\
$\mathbf{6 2 . 6}$ & 69.3 & 66.1 & 64.1 & $\mathbf{2 8 3 . 5}$ & 298.6 & 290.9 & 287.3 & $\mathbf{6 6 8 . 4}$ & 691.7 & 678.2 & 673.7 \\
$\mathbf{3 0 . 5}$ & 33.4 & 32.0 & 31.4 & $\mathbf{1 4 1 . 9}$ & 149.3 & 145.1 & 143.9 & $\mathbf{3 3 4 . 5}$ & 345.7 & 339.6 & 337.3
\end{tabular}

\begin{tabular}{r|r|r|r||r|r|r|r}
\multicolumn{1}{c||}{$80 \times 80$} & \multicolumn{4}{|c}{$100 \times 100$} \\
\hline \multicolumn{1}{c|}{ SWI } & COL & \multicolumn{1}{c||}{ CP1 } & \multicolumn{1}{|c|}{ CP2 } & SWI & COL & \multicolumn{1}{c}{ CP1 } & \multicolumn{1}{c}{ CP2 } \\
\hline 4596.0 & 4554.4 & 4139.5 & $\mathbf{4 0 3 9 . 0}$ & 7587.4 & 7460.6 & 6892.0 & $\mathbf{6 7 6 4 . 5}$ \\
4636.3 & 4585.2 & 4352.7 & $\mathbf{4 2 8 2 . 3}$ & 7459.6 & 7358.1 & 7055.4 & $\mathbf{6 9 5 1 . 2}$ \\
4210.8 & 4183.2 & 4024.6 & $\mathbf{3 9 7 0 . 5}$ & 6706.9 & 6631.8 & 6441.8 & $\mathbf{6 3 7 7 . 5}$ \\
3675.5 & 3653.4 & 3550.4 & $\mathbf{3 5 1 7 . 1}$ & 5819.4 & 5766.7 & 5632.9 & $\mathbf{5 5 9 2 . 6}$ \\
3069.4 & 3071.0 & 3000.4 & $\mathbf{2 9 7 7 . 6}$ & 4874.9 & 4846.1 & 4752.6 & $\mathbf{4 7 2 4 . 3}$ \\
2456.0 & 2473.6 & 2425.1 & $\mathbf{2 4 1 0 . 6}$ & 3899.1 & 3895.1 & 3834.3 & $\mathbf{3 8 1 5 . 0}$ \\
1828.2 & 1864.4 & 1832.3 & $\mathbf{1 8 2 2 . 1}$ & 2915.3 & 2932.5 & 2890.5 & $\mathbf{2 8 7 9 . 8}$ \\
$\mathbf{1 2 1 5 . 2}$ & 1244.6 & 1224.6 & 1220.5 & $\mathbf{1 9 2 2 . 9}$ & 1957.6 & 1934.5 & 1926.4 \\
$\mathbf{6 0 7 . 1}$ & 622.2 & 613.1 & 611.1 & $\mathbf{9 6 0 . 0}$ & 978.5 & 968.1 & 965.1
\end{tabular}

Table 2. Average running time of Switch and ColPerm2 in seconds

\begin{tabular}{|c|c|c|c|c|c|c|c|c|c|}
\hline & & $40>$ & 40 & $60 \times$ & 60 & 80 & 80 & \multicolumn{2}{|c|}{$100 \times 100$} \\
\hline$\overline{\text { SWI }}$ & CP2 & SWI & CP2 & SWI & CP2 & SWI & CP2 & SWI & CP2 \\
\hline$\overline{0.094}$ & 0.084 & 2.361 & 0.516 & 15.111 & 2.921 & 52.522 & 9.383 & 32.550 & 24.700 \\
\hline 0.14 & 045 & 3.08 & 0.483 & 17.112 & 2.445 & 55.919 & 7.85 & 139.931 & 20.639 \\
\hline 0.156 & 1 & 3.1 & 0 & 03 & 2.188 & 75 & 7.8 & 07 & 7.371 \\
\hline 0.149 & 0.038 & 3.080 & 0.369 & 16.010 & 1.877 & 51.955 & 5.951 & 205 & 15.130 \\
\hline 0.1 & 0.033 & 2.776 & 0.332 & 15.147 & 1.623 & 46.227 & 4.8 & 114.348 & 12.227 \\
\hline 0.13 & 27 & 2.38 & 57 & 12.329 & 1.267 & 39.843 & 3.8 & 98.638 & 10.162 \\
\hline 0.1 & & 1.83 & 27 & 9.817 & 0.998 & 31.580 & 3.2 & 335 & 7.664 \\
\hline 0.08 & 0.018 & 1.32 & 0.169 & 6.994 & 0.710 & 22.027 & 2.203 & 53.989 & 5.330 \\
\hline 0.041 & .015 & 0.68 & .098 & 3.778 & 0.438 & 11.550 & 1.288 & 28.745 & 3.053 \\
\hline
\end{tabular}

Table 1 shows the number of 0-1 flips provided by each algorithm. From top to bottom the rows represent the density of the $1 \mathrm{~s}$ in the matrix, from $10 \%$ to $90 \%$. The numerical entries are the averaged result on the 50 matrices for the given size and density. The smallest numbers are typeset in bold. In a similar fashion, Table 2 shows the average running time of SwITCH and ColPERM2 which provided the best values from the viewpoint of optimality. Columns processed the result in less than 0.002 seconds for all matrices, while CoLPERm1 
had a running time of 0.344 seconds in the slowest case. From the tables we deduce that searching through column permutations yields a result much faster than searching through switching components, as SwITCH does. Furthermore, COLPERM2 gave better results for the number of 0-1 flips, especially when the matrix was big and sparse. We suspect that SWITCH performs better when the matrix is small and/or dense, and thus the number of switching components is small. However, this phenomenon should be investigated in more detail.

\section{Conclusion}

We showed that the NP-complete problem of finding the minimal number of $0-1$ flips to make a binary matrix switching component free is equivalent to finding a proper permutation of the columns. Based on this, we designed heuristics, and compared them experimentally to previously published ones. We found that our column based heuristics performed significantly faster and gave better results, in the average case, than the switching component based ones. Due to space considerations, we only presented here test on random matrices, although we observed our method to work pretty well on practical databases, too. We will report on this in a later work. We plan to design more sophisticated heuristic algorithms, by analyzing the cases where our methods fail to give an optimal solution. We also will investigate the case when both 0-1 and 1-0 flips are allowed to make the matrix switching component free.

\section{References}

1. Berthold, M.R., Borgelt, C., Höppner, F., Klawonn, F.: Guide to Intelligent Data Analysis. Springer (2010)

2. Chang, S.K.: The reconstruction of binary patterns from their projections. Comm. ACM 14, 21-25 (1971)

3. Gonzalez, R.C., Woods, R.E.: Digital Image Processing, 3rd edn. Prentice Hall (2008)

4. Herman, G.T., Kuba, A. (eds.): Advances in Discrete Tomography and its Applications. Birkhäuser, Boston (2007)

5. Mannila, H., Terzi, E.: Nestedness and segmented nestedness. In: Proceedings of the 13th ACM SIGKDD International Conference on Knowledge Discovery and Data Mining, KDD 2007, pp. 480-489 (2007)

6. Mitchell, T.M.: Machine Learning. McGraw Hill (1997)

7. Ryser, H.J.: Combinatorial properties of matrices of zeros and ones. Canad. J. Math. 9, 371-377 (1957)

8. Wu, X., Kumar, V. (eds.): The Top Ten Algorithms in Data Mining. Chapman \& Hall/CRC (2007) 